\title{
Efecto hemodinámico esplácnico de somatostatina y octreótido en cirróticos. Estudio con ultrasonografía Doppler
}

\author{
F. J. Fernández Pérez, M. Jiménez Sáenz ${ }^{1}$, J. M. García Montes ${ }^{1}$, J. Rebollo Bernárdez ${ }^{1}$ \\ y J. M. Herrerías Gutiérrez \\ Unidad de Aparato Digestivo. Hospital Costa del Sol. Marbella, Málaga. 'Sección de Hepatología. Servicio de Aparato \\ Digestivo. Hospital Virgen Macarena. Sevilla
}

\section{RESUMEN}

Objetivo: valoración ultrasonografica Doppler del efecto hemodinámico de la administración intravenosa de somatostatina y octreótido.

Material y método: aleatorizamos a 45 cirróticos con varices esofágicas para recibir en una hora una infusión intravenosa de somatostatina (SOM, $250 \mu \mathrm{g}$ ), octreotido (OCT, $50 \mu \mathrm{g}$ ) o placebo (PLA). Pretratamiento y a $15,30,45$ y 60 minutos medimos velocidad media, índice de congestión, volumen de flujo y diámetro de la vena porta además del índice de resistencia en arteria mesentérica superior. Analizamos las concentraciones séricas de bradicinina y péptido intestinal vasoactivo (VIP) en situación basal y a 30 y 60 minutos.

Resultados: respecto de los valores basales tanto SOM como OCT provocaron un descenso significativo en la velocidad $(-19,41$ vs. $-11.19 \%)$ y flujo portal $(-22,79$ vs. $-12,33 \%)$, con aumento del índice de congestión $(+17,5$ vs. $+7,5 \%)$ y del índice de resistencia arterial $(+7,18$ vs. $+6,16 \%)$ respecto de sus valores basales $(p<$ $0,05)$. Estos efectos eran ya evidentes a los 15 minutos y se mantuvieron durante todo el tiempo del estudio. Los cambios inducidos en la velocidad y flujo portal eran más pronunciados con SOM que con OCT, sin diferencias en el índice de congestión o en el índice de resistencia arterial. Los niveles plasmáticos de bradicinina y VIP no experimentaron cambios respecto de sus niveles pretratamiento en ninguno de los grupos.

Conclusiones: a dosis terapeúticas, somatostatina y octreotido reducen la velocidad y flujo portales, incrementando el índice de congestión y el índice de resistencia en la arteria mesentérica superior. La somatostatina determina una reducción del flujo venoso portal más marcada que la inducida por octreótido a pesar de un efecto similar sobre la resistencia arterial esplácnica.

Palabras clave: Hipertensión portal. Cirrosis hepática. Ultrasonografía Doppler. Somatostatina. Octreótido. Péptido intestinal vasoactivo (VIP). Bradicinina.

\begin{abstract}
Aim: Doppler-ultrasound assessment of the splanchnic hemodynamic effects of intravenous somatostatin and octreotide administration.

Material and method: forty-five cirrhotic patients with esophageal varices were randomized to receive 1-hour intravenous somatostatin (SOM, $250 \mu \mathrm{g})$, octreotide (OCT, $50 \mu \mathrm{g})$, or placebo (PLA). In baseline and at 15, 30, 45 and 60 minutes of infusion, mean velocity, congestion index, flow volume and diameter of the portal vein, as well as the superior mesenteric artery resistivity index, were measured. Plasma bradykinine and vasoactive intestinal peptide (VIP) concentrations were also measured at baseline and at 30 and 60 minutes.

Results: while placebo caused no changes in any of the venous and arterial parameters, SOM and OCT caused a sustained decrease in portal vein velocity $(-19.41$ vs. $-11.19 \%)$ and flow $(-22.79$ vs. $-12.33 \%)$, and an increase in the congestion index (+17.5 vs. $+7.5 \%)$ and resistivity index of the superior mesenteric artery $(+7.18$ vs. $+6.16 \%)$ from baseline $(p<0.05)$. These changes were already evident at 15 minutes and remained unchanged over the study period. With respect to OCT, SOM caused a higher reduction in mean velocity and flow in the portal vein, with no significant differences for congestion index and mesenteric artery resistivity index, both increased by SOM and OCT. Plasma bradykinine and VIP concentrations remained unchanged in the three groups.

Conclusions: at therapeutic doses, intravenous somatostatin and octreotide reduce portal vein velocity and flow, and increase portal vein congestion index and superior mesenteric artery resistivity index. Somatostatin causes a higher portal flow reduction than octreotide in spite of a similar splanchnic arterial effect.
\end{abstract}

Key words: Portal hypertension. Liver cirrhosis. Doppler ultrasonography. Somatostatin. Octreotide. Vasoactive intestinal peptide (VIP). Bradykinine.

Fernández Pérez FJ, Jiménez Sáenz M, García Montes JM, Rebollo Bernárdez J, Herrerías Gutiérrez JM. Efecto hemodinámico esplácnico de somatostatina y octreótido en cirróticos. Estudio con ultrasonografía Doppler. Rev Esp Enferm Dig 2008; 100: 552-559. 


\section{INTRODUCCIÓN}

La hemorragia por rotura de varices esofagogástricas puede ser controlada mediante la aplicación de diversos tratamientos como taponamiento esofágico, esclerosis o ligadura endoscópica de varices, establecimiento de shunts portosistémicos quirúrgicos o percutáneos o mediante tratamientos farmacológicos. Estos últimos actúan reduciendo el flujo portal y portocolateral con consecuente reducción de las presiones portal e intravaricosa. Diversos fármacos han sido empleados con este propósito, incluyendo la vasopresina (con o sin nitratos), terlipresina, somatostatina, octreótido y lanreótido. Aunque sólo la terlipresina se ha demostrado capaz de reducir la mortalidad tras una hemorragia aguda varicosa (1), diversos estudios y metaanálisis han demostrado también que estos fármacos son más eficaces que el placebo en el control de la hemorragia por varices (2-4). Además, comparados con el taponamiento esofágico o el tratamiento endoscópico, los fármacos muestran una eficacia similar en el control del sangrado con unos efectos adversos significativamente menores (5-7)

La mayoría de los estudios clínicos y hemodinámicos demuestran que la somatostatina presenta un efecto beneficioso en el control del sangrado varicoso y en la reducción de la presión portal y varicosa (8), si bien en relación al octreótido la evidencia disponible es contradictoria y su posible efecto beneficioso sigue estando aún en controversia. Tampoco queda claro cómo estos fármacos ejercen su efecto, que necesariamente ha de interferir con la vasodilatación esplácnica característica de estos pacientes (9-12).

Aunque se ha propuesto al glucagón como mediador plasmático de somatostatina y octreótido al demostrarse que la administración de estos reduce los niveles plasmáticos de glucagón por inhibición de su síntesis (13), no queda claro qué papel puedan jugar como intermediarios moléculas que también presentan acción vasodilatadora y niveles elevados en sujetos con hipertensión portal, tales como el óxido nítrico, prostaciclinas y péptido intestinal vasoactivo-VIP (11-16). En concreto es muy poco lo que sabemos sobre el papel que puedan ejercer péptidos como el VIP, vasodilatador esplácnico con secuencia de aminoácidos similar al glucagón y que presenta concentraciones elevadas tanto en cirrosis experimental como en humanos (17-20), o como la bradicinina, potente vasodilatador y estímulo para la liberación de óxido nítrico que tan notable importancia tiene en los fenómenos microvasculares del cirrótico.

Nuestro estudio se diseñó para describir el efecto hemodinámico esplácnico de una infusión endovenosa continua de somatostatina y octreótido en pacientes cirróticos con hipertensión portal y valorar el posible papel de bradicinina y VIP como mediadores del efecto vascular de estos fármacos. Hemos empleado las dosis comúnmente administradas en la práctica clínica diaria y la ultrasonografía dúplex-Doppler como procedimiento para la evaluación no invasiva del flujo sanguíneo esplácnico, así como técnicas de RIA (radioinmunoensayo) para la medición de péptidos vasoactivos.

\section{MATERIAL Y MÉTODO}

\section{Grupos de estudio y protocolo}

Cincuenta y cinco pacientes cirróticos con hipertensión portal y varices esofagogástricas diagnosticadas por endoscopia fueron considerados candidatos para este estudio. Doce de ellos fueron excluidos (Tabla I). Los 45 pacientes restantes, 14 mujeres y 31 varones, con edad media de 55,4 años (rango: 45-65) fueron aleatorizados mediante tablas de asignación aleatoria en 3 grupos de 15 pacientes cada uno. El grupo placebo (PLA) recibió una infusión continua de $100 \mathrm{ml}$ de salino $0,9 \%$ durante 1 hora. El grupo somatostatina (SOM) recibió $250 \mu \mathrm{g}$ de somatostatina (Serono Laboratories, Switzerland) en $100 \mathrm{ml}$ de salino $0,9 \%$ durante 1 hora. El grupo octreótido (OCT) recibió una infusión continua de $50 \mu \mathrm{g}$ de octreótido (Serono Laboratories, Switzerland) en $100 \mathrm{ml}$ de solución salina $0,9 \%$ durante 1 hora.

\section{Tabla I. Criterios de exclusión}

1. Edad $<18$ años o $>75$ años

2. Insuficiencia hepática grave (actividad de protrombina $<40 \%$ con o sin encefalopatía hepática)

3. Portador de shunt quirúrgico portosistémico

4. Visualización inadecuada de los vasos esplácnicos por ultrasonografía en modo B

5. Trombosis portal

6. Vena paraumbilical permeable

7. Enfermedad sistémica descompensada

8. Administración de fármacos vasoactivos en las 24 horas previas a ser administradas somatostatina u octreótido

9. Hipersensibilidad conocida a los fármacos usados en el estudio

\section{Mediciones}

Para el análisis no invasivo del efecto vascular esplácnico de somatostatina y octreótido empleamos un ecógrafo con Doppler color y pulsado con transductor sectorial electrónico de 3,5 MHz (Hitachi EUB 515, Tokio-Japón). Las mediciones en la vena porta se realizaron mediante abordaje subcostal en el punto de cruce de la porta con la arteria hepática, con ángulo Doppler menor a $60^{\circ}$. Las mediciones en la arteria mesentérica superior se realizaron en un punto $2 \mathrm{~cm}$ distal al origen de dicha arteria en la aorta. Los valores obtenidos para cada parámetro y en cada momento de medición fueron el resultado de obtener la media de 3 mediciones consecutivas. Todo el estudio Doppler fue llevado a cabo por un único explorador con experiencia en ecografía Doppler, 
sin conocimiento sobre a qué grupo estaba asignado cada paciente.

Tras un ayuno de al menos 8 horas y permanecer 30 minutos en supino se practicaron mediciones en situación basal (previa a la administración de fármacos) y a los 15 , 30, 45 y 60 minutos de infusión. Se midieron el diámetro de la vena porta (DVP), la velocidad media portal (VVP), volumen de flujo portal (FVP), índice de congestión en vena porta (ICVP) e índice de resistencia de la arteria mesentérica superior (IRAMS). Se calculó el FVP (VVP $\mathrm{x}$ área de sección portal), ICVP (área de sección portal/ VVP) e IRAMS ([Vmax-Vmin]/Vmin), considerando circulares los vasos. Adicionalmente medimos en situación basal y a los $15,30,45$ y 60 minutos del inicio de administración de fármacos la presión arterial media (PAM: [sistólica+diastólica x 2]/3), frecuencia cardiaca (FC) y los niveles plasmáticos de glucosa.

En cada grupo los valores basales de cada parámetro hemodinámico estudiado fueron comparados con los obtenidos en los distintos momentos de medición durante la infusión de fármacos. Los cambios absolutos y relativos de los mismos fueron comparados entre los 3 grupos de pacientes.

Los niveles séricos de bradicinina y VIP fueron analizados en situación basal y a los 30 y 60 minutos de infusion intravenosa de fármacos mediante técnica de radioinmunoensayo (RIA, ICN Pharmaceuticals, Costa Mesa, California, EE. UU.) de muestras de suero de los pacientes congeladas tras centrifugación a $-40^{\circ}$ centígrados.

\section{Estadísticas}

Empleamos el paquete SPSS-10 (Statistical Package for Social Sciences, Chicago, IL) para el análisis estadístico. Los resultados se expresan en su valor medio y desviación estándar. Se empleó el test de Kolgomorov-Smirnov para verificar el carácter de distribución normal de los diferentes parámetros medidos. Para variables cuantitativas empleamos el test de t-Student y test ANOVA, mientras que para variables cualitativas empleamos el test de Chi cuadrado y el test exacto de Fisher. Se consideró significación estadística para valores de la p $<0,05$.

\section{Consideraciones éticas}

El protocolo de estudio fue diseñado de acuerdo con las consideraciones éticas de la Declaración de Helsinki de 1975 y fue aprobado por el Comité Local de Ética del Hospital. Todos los pacientes firmaron el correspondiente consentimiento informado a la inclusión en el estudio.

\section{RESULTADOS}

Los datos clínicos y biológicos de los pacientes incluidos en el presente estudio se resumen en la tabla II. No se
Tabla II. Características epidemiológicas, clínicas y biológicas de los pacientes incluidos en los tres grupos de estudio

\begin{tabular}{lcccc}
\hline & Octreótido & Somatostatina & Placebo & $p$ \\
\hline Edad (años) & $55,8 \pm 11,7$ & $55,4 \pm 9,8$ & $56 \pm 7,6$ & $\mathrm{~ns}$ \\
Sexo (hombre/mujer) & $9: 6$ & $10: 5$ & $11: 4$ & $\mathrm{~ns}$ \\
Child-Pugh & $6,3 \pm 1,2$ & $7,8 \pm 2,3$ & $7,9 \pm 2,8$ & $\mathrm{~ns}$ \\
Etiología & & & & \\
$\quad$ Alcohol & 4 & 11 & 10 & \\
$\quad$ Virus & 6 & 2 & 3 & \\
$\quad$ Otras & 5 & 2 & 2 & \\
Bilirrubina (mg/dl) & $1,3 \pm 0,8$ & $2,1 \pm 1,7$ & $2,1 \pm 1,2$ & $\mathrm{~ns}$ \\
ALT(U/l) & $61,5 \pm 59,9$ & $86,2 \pm 87,4$ & $49,4 \pm 35,4$ & $\mathrm{~ns}$ \\
Protrombina (s) & $13,8 \pm 1,9$ & $15,6 \pm 2,3$ & $15,1 \pm 2,7$ & $\mathrm{~ns}$ \\
Albúmina (g/dl) & $3,7 \pm 0,3$ & $3,7 \pm 0,9$ & $3,5 \pm 0,6$ & $\mathrm{~ns}$ \\
Creatinina (mg/dl) & $0,7 \pm 0,1$ & $0,9 \pm 0,6$ & $0,8 \pm 0,5$ & $\mathrm{~ns}$ \\
Ascitis & 9 & 10 & 9 & $\mathrm{~ns}$ \\
HDA-VEG & 6 & 9 & 6 & $\mathrm{~ns}$ \\
EVE/LEV & 4 & 7 & 5 & $\mathrm{~ns}$ \\
Colaterales & 4 & 3 & 6 & $\mathrm{~ns}$ \\
\hline
\end{tabular}

HDA-VEG: episodio previo de hemorragia digestiva alta por varices esofagogástricas; EVE/LEV: esclerosis de varices esofágicas/ligadura endoscópica de varices con anterioridad; Colaterales: incluye shunts venosos de descompresión a retroperitoneo, esplenorrenales, vena gástrica izquierda o en territorio de venas gástricas cortas; ns: ausencia de diferencias estadísticamente significativas.

apreciaron en situación basal diferencias estadísticamente significativas en las características epidemiológicas, bioquímicas, grado de Child-Pugh, frecuencia cardiaca o presión arterial media entre los 3 grupos de estudio.

No hubo cambios significativos en ninguno de los 3 grupos de estudio y a lo largo del tiempo de administración de fármacos en los valores de presión arterial media, frecuencia cardiaca o glucosa plasmática y tampoco se hallaron cambios significativos en el diámetro de la vena porta durante la administración de placebo, somatostatina u octreótico, lo que supone que el área de sección portal no sufrió cambios aun con la administración de sustancias (área $=\pi \cdot r^{2}$ ).

Mientras que en el grupo placebo (PLA) no produjeron cambios significativos en VVP, FVP, ICVP y IRAMS respecto de los valores basales, tanto en el grupo somatostatina (SOM) como en el octreótido (OCT) se evidenció un descenso significativo de la VVP ya en el primer punto de medición (15 minutos) respecto de los valores basales. De forma similar se observó un descenso significativo de FVP a los 15 minutos durante la administración de somatostatina y octreótido, como muestra la tabla III. Estos cambios en VVP y FVP ya evidentes en la medición de los 15 minutos, se mantuvieron a lo largo del tiempo del estudio. El descenso medio de la VVP alcanzó un $19,41 \%$ en el grupo-SOM y un $11,13 \%$ en el grupoOCT, mientras que la reducción media del FVP fue del $22,79 \%$ en el grupo-SOM frente al $12,33 \%$ en el grupoOCT.

Por efecto de la administración de somatostatina el ICVP alcanzó en el primer momento de medición (15 minutos) un incremento significativo respecto a sus valores basales, manteniéndose este efecto a todo lo largo del 
Tabla III. Efectos de la administración intravenosa

\begin{tabular}{|c|c|c|c|c|c|}
\hline & Basal & $15 \mathrm{~min}$ & $30 \mathrm{~min}$ & $45 \mathrm{~min}$ & $60 \mathrm{~min}$ \\
\hline $\begin{array}{l}\text { WV }(\mathrm{cm} / \mathrm{s}) \\
\text { Placebo } \\
\text { Somatostatina } \\
\text { Octreótido }\end{array}$ & $\begin{array}{l}14,63 \pm 4,17 \\
13,99 \pm 2,03 \\
14,02 \pm 2,75\end{array}$ & $\begin{array}{c}14,6 \pm 4,21 \\
11,35 \pm 2,08^{*} \\
12,81 \pm 2,07^{*}\end{array}$ & $\begin{array}{c}14,8 \pm 4,37 \\
11,37 \pm 1,75^{*} \\
12,38 \pm 2,15^{*}\end{array}$ & $\begin{array}{c}14,6 \pm 4,1 \\
11,33 \pm 1,74^{*} \\
12,34 \pm 2,82^{*}\end{array}$ & $\begin{array}{c}14,64 \pm 3,88 \\
11,05 \pm 1,71 * \\
12,34 \pm 2,57 *\end{array}$ \\
\hline $\begin{array}{l}\text { FVP }(\mathrm{m} / / \mathrm{min}) \\
\text { Placebo } \\
\text { Somatostatina } \\
\text { Octreótido }\end{array}$ & $\begin{array}{c}1.110,86 \pm 421,53 \\
976,41 \pm 238,71 \\
1.231,85 \pm 465,14\end{array}$ & $\begin{array}{c}1.075,78 \pm 418,20 \\
768,32 \pm 264,66^{*} \\
1.109,84 \pm 447,35^{*}\end{array}$ & $\begin{array}{c}1.133,15 \pm 488,21 \\
740,66 \pm 184,02^{*} \\
1.090,11 \pm 426,38^{*}\end{array}$ & $\begin{array}{c}1.084,31 \pm 428,72 \\
747,01 \pm 236,68^{*} \\
1.065,84 \pm 427,58^{*}\end{array}$ & $\begin{array}{c}1.100,47 \pm 428,54 \\
759,69 \pm 242,87 * \\
1.054,20 \pm 436,76^{*}\end{array}$ \\
\hline $\begin{array}{l}\text { ICVP (cm/s) } \\
\text { Placebo } \\
\text { Somatostatina } \\
\text { Octreótido }\end{array}$ & $\begin{array}{l}0,093 \pm 0,040 \\
0,085 \pm 0,027 \\
0,105 \pm 0,043\end{array}$ & $\begin{array}{c}0,089 \pm 0,035 \\
0,101 \pm 0,029^{* *} \\
0,109 \pm 0,039\end{array}$ & $\begin{array}{c}0,091 \pm 0,036 \\
0,099 \pm 0,029^{*} \\
0,115 \pm 0,040^{*}\end{array}$ & $\begin{array}{c}0,089 \pm 0,036 \\
0,099 \pm 0,026^{* *} \\
0,115 \pm 0,038^{*}\end{array}$ & $\begin{array}{c}0,088 \pm 0,032 \\
0,108 \pm 0,027^{*} \\
0,115 \pm 0,039^{*}\end{array}$ \\
\hline $\begin{array}{l}\text { IRAMS } \\
\text { Placebo } \\
\text { Somatostatina } \\
\text { Octreótido }\end{array}$ & $\begin{array}{l}0,820 \pm 0,041 \\
0,808 \pm 0,053 \\
0,813 \pm 0,028\end{array}$ & $\begin{array}{c}0,823 \pm 0,042 \\
0,831 \pm 0,072 \\
0,855 \pm 0,019 * *\end{array}$ & $\begin{array}{c}0,831 \pm 0,031 \\
0,865 \pm 0,049^{*} \\
0,872 \pm 0,021 *\end{array}$ & $\begin{array}{c}0,832 \pm 0,034 \\
0,873 \pm 0,053^{*} \\
0,876 \pm 0,017^{*}\end{array}$ & $\begin{array}{c}0,840 \pm 0,036 \\
0,877 \pm 0,047^{\star} \\
0,876 \pm 0,016^{\star}\end{array}$ \\
\hline $\begin{array}{l}\text { Péptidos vasoactiv } \\
\text { VIP (pmol/ml) } \\
\text { PLA } \\
\text { SOM } \\
\text { OCT }\end{array}$ & $\begin{array}{l}3,09 \pm 0,64 \\
3,44 \pm 0,55 \\
3,53 \pm 0,81\end{array}$ & & $\begin{array}{l}3,47 \pm 1,15 \\
3,16 \pm 0,53 \\
3,43 \pm 1,04\end{array}$ & & $\begin{array}{l}3,62 \pm 0,74 \\
3,01 \pm 1,66 \\
3,67 \pm 0,55\end{array}$ \\
\hline $\begin{array}{l}\text { Bradicinina (pm } \\
\text { PLA } \\
\text { SOM } \\
\text { OCT }\end{array}$ & $\begin{array}{l}706,13 \pm 280,75 \\
818,56 \pm 461,79 \\
743,79 \pm 507,30\end{array}$ & & $\begin{array}{l}617,84 \pm 371,87 \\
546,97 \pm 399,64 \\
621,21 \pm 359,20\end{array}$ & & $\begin{array}{c}765,1 \pm 374,22 \\
735,25 \pm 300,34 \\
749,44 \pm 334,01\end{array}$ \\
\hline
\end{tabular}

Efectos de la administración intravenosa de placebo, somatostatina y octreótido sobre la velocidad de la sangre en la vena porta (VVP), volumen de flujo sanguíneo portal (FVP), índice de congestión venoso portal (ICVP), índice de resistencia de la arteria mesentérica superior (IRAMS) y niveles plasmáticos de péptidos vasoactivos. Los valores se expresan como media \pm desviación estándar. ${ }^{*} p<0,01$ y * $p<0,05$ respecto de la situación basal.

tiempo de estudio. Una situación similar fue observada en el grupo-OCT, si bien el incremento del ICVP no alcanzó significación estadística hasta la medición de los 30 minutos. El incremento medio del ICVP fue del 17.05 y 7,5\% para los grupos SOM y OCT, respectivamente.

Por efecto de la administración de fármacos, el IRAMS se incrementó en ambos grupos SOM y OCT comparados con sus correspondientes valores basales. Mientras que en el grupo-OCT este incremento alcanzó significación estadística ya en la medición de los 15 minutos, en el grupo-SOM dicha significación estadística fue alcanzada por primera vez en la medición de los 30 minutos. Estos cambios inducidos por somatostatina y octreótido en el IRAMS se mantuvieron hasta el final del periodo de estudio sin cambios significativos (Tabla III). El incremento medio del IRAMS fue del 7,18\% con somatostatina y del $6,16 \%$ con octreótido.

Para facilitar la comparabilidad entre los grupos SOM y OCT valoramos los cambios relativos en los parámetros ultrasonográficos de hemodinámica esplácnica secundarios a la administración de drogas vasoactivas, entendidos como la diferencia entre los valores basales y los obtenidos a los $15,30,45$ y 60 minutos. Con este análisis los cambios relativos en la VVP del grupo SOM se demostraron significativamente mayores que los observados en el grupo OCT a los 15 y 60 minutos ( $\mathrm{p}=0,017$ y $\mathrm{p}=0,019$ respectivamente), mientras que no se apreciaron diferencias significativas a los 30 y 45 minutos de infusión ( $\mathrm{p}=0,123$ y $\mathrm{p}=0,082$ respectivamente). Sin embargo, los cambios medios (antes/durante la administración de fármacos) mostraban que la somatostatina era más eficaz que el octreótido en reducir la VVP $(19,41 v s .11,13 \%$ respectivamente, $\mathrm{p}=$ $0,015)$. De forma similar, los cambios relativos en FVP fueron más intensos con somatostatina que con octreótido a los 15 y 30 minutos ( $\mathrm{p}=0,026$ y $\mathrm{p}=0,045$ respectivamente), sin cambios estadísticamente significativos a los 45 y 60 minutos $(\mathrm{p}=0,112$ y $\mathrm{p}=0,08$ respectivamente). Cuando se consideró el efecto farmacológico medio (antes/durante la administración de fármacos) la reducción de FVP inducido por somatostatina fue significativamente mayor que la lograda con octreótido $(22,79$ vs. $12,33 \%, \mathrm{p}=0,005)$. Por el contrario, no se apreciaron diferencias significativas entre los grupos SOM y OCT al valorar los cambos relativos en ICVP e IRAMS (Figs. 1 y 2).

Con respecto a los péptidos vasoactivos, nuestros datos muestran que ni ante la administración de placebo, somatostatina u octreótido se producen cambios significativos en las concentraciones plasmáticas de VIP o bradicinina respecto de sus valores pretratamiento, como muestra la tabla III. 
VVP

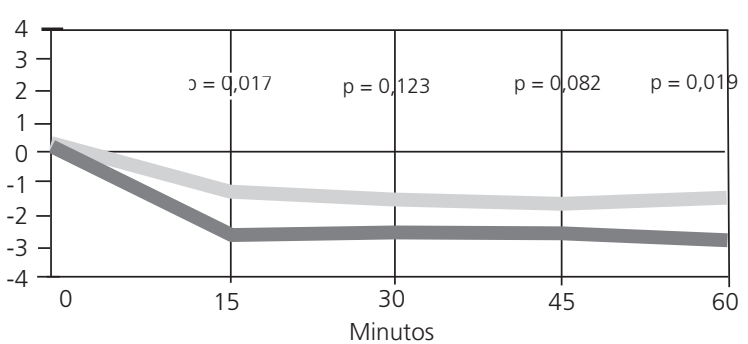

FVP

- Somatostatina $=$ Octreótido

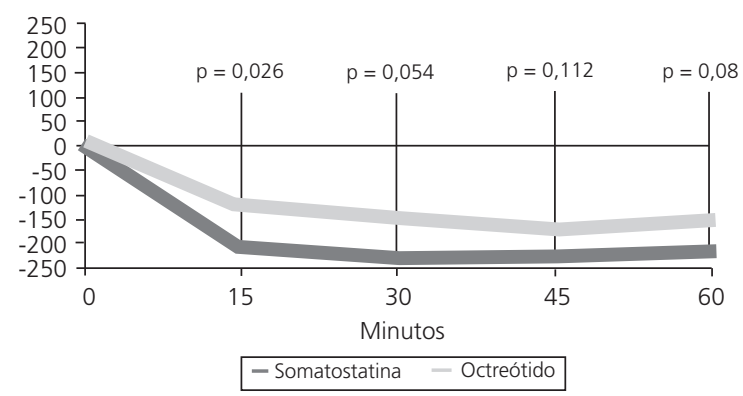

ICVP
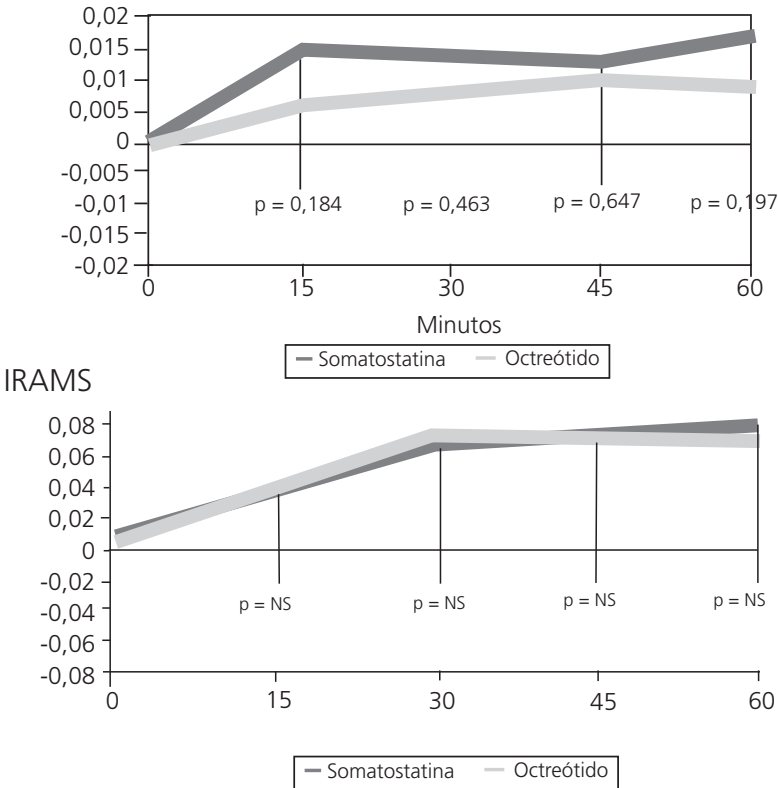

Fig. 1. Comparación de los cambios relativos en VVP, FVP, ICVP e IRAMS durante la administración intravenosa de somatostatina (SOM) y octreótido $(\mathrm{OCT})$.

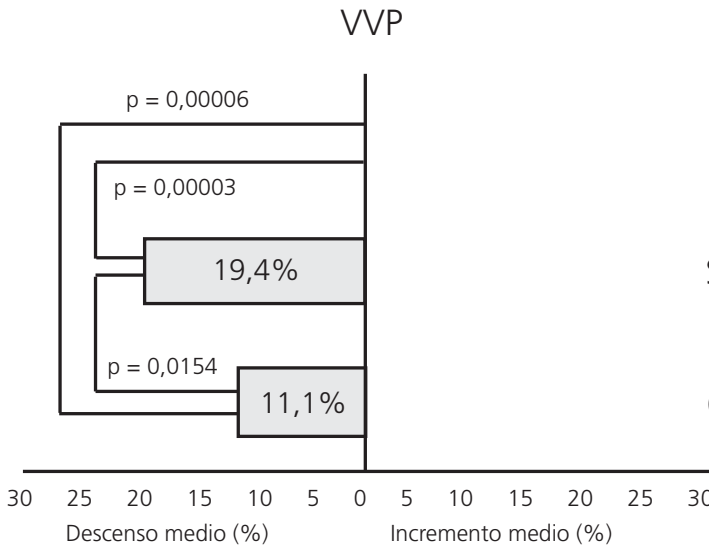

FVP

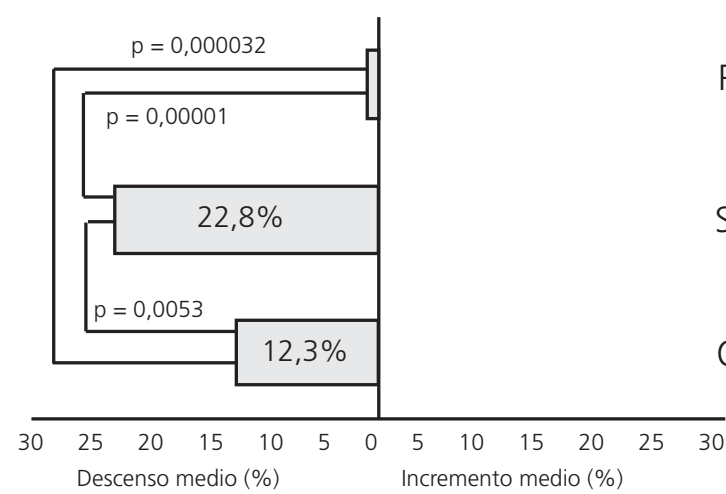

PLA

SOM

OCT

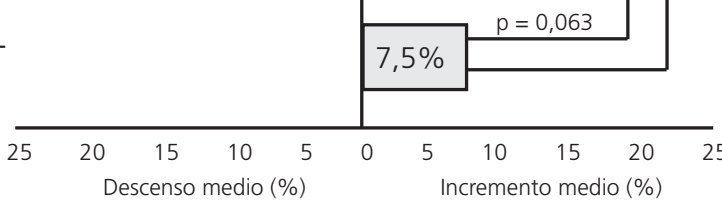

IRAMS

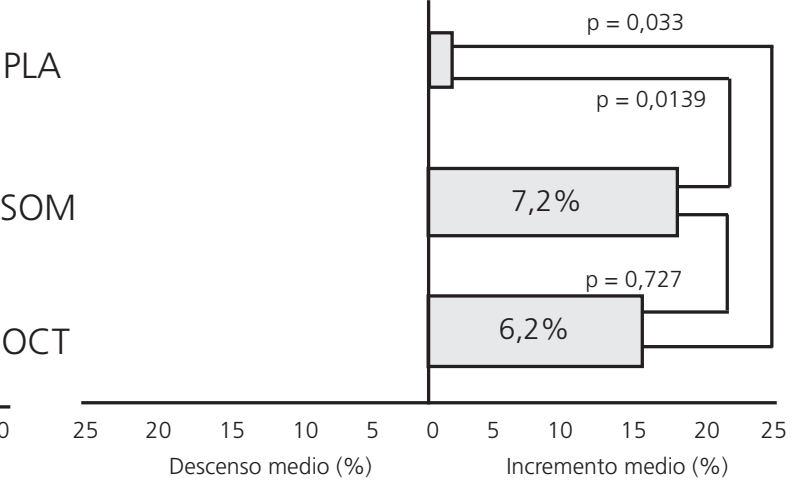

Fig. 2. Cambios medios en VVP, FVP, ICVP e IRAMS durante la administración de placebo (PLA), somatostatina (SOM) y octreótido (OCT). 


\section{DISCUSIÓN}

Diversos estudios clínicos y hemodinámicos han demostrado un efecto beneficioso de la somatostatina en el control del sangrado por varices esofágicas y en la reducción de la presión portal y portocolateral. La evidencia disponible al respecto de octreótido o de los mecanismos de acción de ambos fármacos es menor. Mientras un metaanálisis concluye en un claro efecto beneficioso del octreótido en el control del sangrado varicoso, diversos estudios hemodinámicos muestran resultados contradictorios en términos de reducción de presión portal, flujo portocolateral y duración de efecto (4,21-29). Además, aunque muchos péptidos plasmáticos se han propuesto como intermediarios plasmáticos de somatostatina y octreótido, no esta aún del todo claro cuáles son y cómo ejercen este papel.

Nuestro estudio fue diseñado para valorar de forma no invasiva (ultrasonografía dúplex-Doppler) el efecto de somatostatina y octreótido sobre la circulación esplácnica en pacientes cirróticos con hipertensión portal, así como el posible papel de ciertos péptidos vasoactivos (péptido intestinal vasoactivo-VIP y bradicinina) como intermediarios plasmáticos del efecto de dichos fármacos. Las dosis y vía de administración son las que se usan en la práctica clínica habitual para el control del sangrado por varices esofágicas. La ultrasonografía Doppler se seleccionó como técnica de evaluación hemodinámica no invasiva, toda vez que diversos estudios han demostrado buena correlación con técnicas invasivas como la medición del gradiente de presión portal, además de su capacidad para detectar cambios agudos en la circulación esplácnica inducidos por fármacos $(26,30-36,46)$.

Aunque algunos autores han informado de bradicardia transitoria tras un bolo intravenoso de $50 \mu \mathrm{g}$ de octreótido $(29,47)$, incremento transitorio de la PAM (29) o reducción de la PAM, FC y gasto cardico tras 60 minutos de una administración intravenosa de somatostatina (28), nuestros pacientes no presentaron ningún cambio significativo en la PAM, FC o glucosa plasmática durante la administración de somatostatina o de octreótido.

Diversos estudios en cirróticos con hipertensión portal han mostrado buena correlación entre el gradiente de presión suprahepática (GSH) y diversos parámetros ecográficos $(31-34,48,49)$. Entre estos la velocidad media en la porta (VVP) y el flujo medio portal (FVP) se consideran por varios autores buenos indicadores ecográficos de los cambios agudos en el lecho vascular esplácnico tras la administración de fármacos (35,36-46).

Nuestros resultados son coincidentes con otros trabajos publicados que describen la ausencia de cambios en el diámetro de la vena porta con el uso de diferentes fármacos y vías de administración tales como porpanolol, dinitrato de isosorbide, octreótido, lanreótido, metoclopramida y vasopresina $(30,50-52)$. Ello parece indicar que ni somatostatina ni octreótido ejercen efectos significativos sobre la pared venosa portal. En consecuencia, si el área de sección de la vena porta no cambia, el flujo de la vena porta es directamente influido por la velocidad de la sangre en el vaso, de acuerdo a la ley de Laplace.

En nuestro estudio tanto somatostatina como octreótido provocan un descenso significativo tanto de VVP como FVP respecto sus valores basales $(p<0,01)$, efecto que se mantiene durante la administración de los mismos y que es más marcado con somatostatina que con octreótido (VVP: 19,41 vs. 11,13\%; p < 0,05; FVP: 22,79 vs. $12,33 \% ; \mathrm{p}<0,01)$. El trabajo de Shiedermaier y cols. (30) sobre cirróticos con TIPS afirmaba que con una perfusión de $25 \mu \mathrm{g} / \mathrm{h}$ de octreótido se obtenía una reducción del FVP del 16,2 $\pm 6,4 \%$ y del $11,7 \pm 4,6 \%$ cuando las dosis se incrementaron a $100 \mu \mathrm{g} / \mathrm{h}$, resultados estos similares a los nuestros con $50 \mu \mathrm{g} / \mathrm{h}$. Otros estudios que también han pretendido aclarar el papel de somatostatina y octreótido en la hemodinámica esplácnica incluyen el de Ludwig y cols. (53), que describía el mantenimiento del efecto del octreótido intravenoso durante al menos 48 horas, o el de Nishida y cols. (29) en el que las mediciones Doppler en vena ácigos tras administración de somatostatina $(250 \mu \mathrm{g} / \mathrm{h}$ previo bolo de $250 \mu \mathrm{g}) \mathrm{u}$ octreótido (50 $\mu \mathrm{g} / \mathrm{h}$ previo bolo de $50 \mu \mathrm{g}$ ) apreciaban un inicial descenso del flujo venoso del 23 y $47 \%$ respectivamente, con un repunte a los 60 minutos del $27 \%$ sólo en los tratados con somatostatina, pero no con octreótido. También es interesante, aunque usando catéteres de presión intravascular como elemento de medida, el estudio de Yang y cols. (27) sobre cirróticos con TIPS y con dosis terapéuticas de somatostatina $(250 \mu \mathrm{g})$ y octreótido $(25 \mu \mathrm{g} / \mathrm{h})$ demostró que ambos fármacos reducían la presión portal, aunque significativamente más la somatostatina que el octreótido, con dosis y vías de administración similares a los usados por nosotros en el presente estudio.

En nuestro estudio también pudimos comprobar que somatostatina y octreótido determinan un claro efecto arterial esplácnico al incrementar el IRAMS respecto de los valores basales, siendo este efecto más precoz con octreótido (a los 15 minutos, $\mathrm{p}<0,01$ ) que con somatostatina (a los 30 minutos, $p<0,01$ ). Este efecto, una vez alcanzado, se mantuvo estable a lo largo del estudio, sin diferencias significativas entre los grupos SOM y OCT (IRAMS: $+7,18 v s .+6,16 \%$ respectivamente, $\mathrm{p}=0,727$ ). Nuestros datos son equiparables con los referidos por Schiedermaier y cols. (30) con octreótido $(+3,1 \pm 1,1$ con $25 \mu \mathrm{g} / \mathrm{h}$ y $+2,9 \pm 0,7 \%$ al subir a $100 \mu \mathrm{g} / \mathrm{h}$ ) o los referidos por Saruc y cols. (54) con somatostatina intravenosa (250 $\mu \mathrm{g} / \mathrm{h}$ ) en pacientes sin hipertensión portal pero con sangrado digestivo alto, en el que se describe un descenso del FVP y un incremento del IRAMS sin cambios en el índice de resistencia de la arteria renal, lo que viene nuevamente a sugerir un efecto selectivo de la somatostatina sobre el territorio esplácnico.

$\mathrm{Si}$ el FVP es reducido tanto por somatostatina como por octreótido sin inducir cambios en el diámetro de la vena porta, este efecto debe depender de una reducción del aflujo venoso o de un incremento del flujo portocola- 
teral. Hasta el momento no hay evidencia clínica o experimental de cambio en las resistencias intrahepáticas por efecto de estos fármacos. Sin embargo hay una evidencia cada vez mayor de un efecto vasoconstrictor de la somatostatina y del octreótido sobre los vasos arteriales esplácnicos. Nuestro estudio demuestra de forma no invasiva que a la par que se produce un descenso del flujo venoso portal, el IRAMS (reflejo de las resistencias arteriales esplácnicas) se incrementa. Además, mientras que tanto la somatostatina $(250 \mu \mathrm{g} / \mathrm{h})$ como el octreótido (50 $\mu \mathrm{g} / \mathrm{h}$ ) muestran un efecto muy similar sobre los vasos arteriales esplácnicos (en nuestro estudio la arteria mesentérica superior), determinan efectos significativamente diferentes en los parámetros de flujo venoso portal. Puesto que el octreótido es un análogo semisintético de la somatostatina y es bien sabida su diferente afinidad por los receptores de somatostatina, estos datos podrían indicar que las diferencias evidenciadas con somatostatina y octreótido podrían ser debidas a un diferente efecto en la circulación portocolateral o sobre la circulación intrahepática.

Respecto de los péptidos plasmáticos, aunque la evidencia acumulada apunta al glucagón como el principal mediador plasmático no derivado del endotelio con efecto vasodilatador (13), muchas otras moléculas (histamina, bradicinina, VIP, óxido nítrico, adenosina, endotelina y prostaciclina, entre otras) se han implicado en el estatus vasodilatado de los pacientes cirróticos. Si además estas moléculas juegan algún papel en la mediación del efecto de somatostatina y octreótido, aún no ha sido aclarada. Nuestros resultados parecen apuntar que al menos ni el VIP ni la bradicinina juegan papel alguno como mediadores plasmáticos del efecto de somatostatina o del octreótido.

Aunque pensamos que nuestro estudio ofrece una información complementaria para la comprensión de los efectos de fármacos vasoactivos sobre la circulación esplácnica en los pacientes cirróticos, creemos que son necesarios más trabajos que permitan clarificar estos aspectos.

\section{BIBLIOGRAFÍA}

1. Ioannou G, Doust J, Rockey DC. Terlipressin for acute esophageal variceal hemorrhage. Cochrane Database Syst Rev 2001; 1: CD002147.

2. Burroughs AK, McCormick PA, Hughes MD, et al. Randomized double-blind placebo-controlled trial of somatostatin for variceal bleeding. Emergency control and prevention of early variceal rebleeding. Gastroenterology 1990; 99 (5): 1388-95.

3. Averignos A, Nevens F, Raptis S, et al. Early administration of somatostatin and efficacy of sclerotherapy in acute esophageal variceal bleeds: the European Bleeding Oesophageal Variceal Episodes (ABOVE) randomized trial. Lancet 1997; 350 (9090): 1495-9.

4. Corley DA, Cello JP, Adkisson W, et al. Octreotide for acute esophageal variceal bleeding: a meta-analysis. Gastroenterology 2001; 120: 946-54.

5. Imperiale TF, Teran JC, McCullough AJ. A meta-analysis of somatostatin versus vasopressin in the management of acute esophageal variceal haemorrhage. Gastroenterology 1995; 109 (4): 1289-94.
6. Feu F, Ruiz del Árbol I, Banares R, et al. Double blind randomized controlled trial comparing terlipressin and somatostatin for acute variceal hemorrhage. Variceal Bleeding Study Group. Gastroenterology 1996; 111 (5): 1291-9.

7. Jaramillo JL, De la Mata M, Miño G, et al. Somatostatin versus Sengstaken balloon tamponade for primary hemostasis of bleeding esophageal varices. A randomized pilot study. J Hepatol 1991; 12 (1): 100-5

8. Cirera I, Feu F, Luca A, et al. Effects of bolus injections and continuous infusions of somatostatin and placebo in patients with cirrhosis: a double-blind hemodynamic investigation. Hepatology 1995; 22 (1): 106-11.

9. Silva G, Navasa M, Bosh J, et al. Hemodynamic effect of glucagon in portal hipertension. Hepatology 1990; 11: 668-73.

10. Pizcueta MP, Casamitjana R, Bosh J, et al. Decreased systemic vascular sensitivity to norepinephrine in portal hypertensive rats. Role of hyperglucagonism. Am J Physiol 1990; 258: 191-5.

11. Sieber CC, López-Talavera JC, Groszmann RJ. Role of nitric oxide in the in vitro splachnic vascular hyporeactivity in ascitic cirrhotic rats. Gastroenterology 1993; 104: 1750-4.

12. Sieber CC, Groszmann RJ. Nitric oxide mediates hyporeactivity to vasopressors in mesenteric vessels of portal hypertensive rats. Gastroenterology 1992; 103: 2354-9.

13. Cirera I, Feu F, Luca M, et al. Effects of bolus injectionsand continuous infusion of somatostatin and placebo in patients with cirrosis: a double-blind hemodynamic investigation. Hepatology 1995; 22 : 106-11.

14. Pak JM, Lee S. Glucagon in portal hypertension. J Hepatol 1994; 20: 825-32.

15. Sherwin RS, Fisher M, Bessoff J, et al. Hyperglucagonemia in cirrhosis: altered secretion and sensitivity to glucagons. Gastroenterology $1978 ; 74: 1224-8$.

16. Sitzmann JV, Li SS, Lin PW, et al. Prostacyclin mediates splachnic vascular response to norepinephrine in portal hypertension. J Surg Res 1989; 47: 208-11.

17. Henriksen JH, Staun-Olsen P, Borg Mogensen N, et al. Circulating endogenous vasoactive intestinal polypeptide (VIP) in patients with uremia and liver cirrhosis. Eur J Clin Invest 1986; 16: 211-6.

18. Geraghty JG, Angerson WJ, Carter DC. Splachnic haemodynamics and vasoactive agents in experimental cirrhosis. HPB Surg 1994; 8: 83-8.

19. Lee SS, Huang M, Ma Z, Rorstad O. Vasoactive intestinal peptide in cirrhotic rats: hemodynamic effects and mesenteric arterial receptor characteristics. Hepatology 1996; 23 (5): 1174-80.

20. Hunt S, Vaamonde CA, Rattassi T, Berian G, Said SI, Papper S. Circulating levels of vasoactive intestinal polypeptide in liver disease. Archives of Internal Medicine 1979; 139 (9): 994-6.

21. Escorsell A, Bandi JC, Andreu V, et al. Desensitization to the effects of intravenous octreotide in cirrhotic patients with portal hypertension. Gastroenterology 2001; 120: 161-9.

22. Moller S, Brinch K, Henriksen JH, et al. Effect of octreotide on systemic, central and splachnic haemodynamics in cirrhosis. J Hepatol 1997; 26 (5): 1026-33.

23. Nevens F, Van Steenbergen W, Yap SH, et al. Assessment of variceal pressure by continuous non-invasive endoscopic registration: a placebo controlled evaluation of the effect of terlipressin and octreotide. Gut 1996; 38 (1): 129-34.

24. McKee R. A study of octreotide in oesophageal varices. Digestion 1990; 45 (Supl. 1): 60-4 (discussion 65).

25. Pringle SD, McKee RF, Garden OJ, et al. The effect of a long-acting somatostatin analogue on portal and systemic haemodynamics in cirrhosis. Aliment Pharmacol Ther 1988; 2 (5): 451-9.

26. Lin HC, Yang YY, Hou MC, et al. Hemodynamic effects of a combination of octreotide and terlipressin in patients with viral hepatitis related cirrhosis. Scand J Gastroenterol 2002; 37 (4): 482-7.

27. Yang J, Wu X, Li J, et al. Effect of somatostatin versus octreotide on portal haemodynamics in patients with cirrhosis and portal hypertension. Eur J Gastroenterol Hepatol 2005; 17 (1): 53-7.

28. Kalambokis G, Economou M, Paraskevi K, et al. Effects of somatostatin, terlipressin and somatostatin plus terlipressin on portal and systemic hemodynamics and renal sodium excretion in patients with cirrosis. J Gastroenterol Hepatol 2005; 20: 1075-81.

29. Nishida H, Giostra E, Spahr L, et al. Valiodation of color Doppler EUS for azygos blood flor measurement in patients with cirrhosis: 
application to the acute hemodynamic effects of somatostatin, octreotide, or placebo. Gastrointest Endoscop 2001; 54 (1): 24-30.

30. Shiedermaier P, Göke B, Sauerbruch T. Effects of different octreotide dosages on splachnic hemodynamics and glucagon in patients with TIPS. Am J Gatroenterol 2001; 96 (7): 2218-24.

31. Taourel P, Blanc P, Dauzat M, et al. Doppler study of mesenteric, hepatic, and portal circulation in alcoholic cirrhosis: relationship between quantitative doppler measurements and the severity of portal hypertension and hepatic failure. Hepatology 1998; 28: 932-6.

32. Merkel C, Sacerdotti D, Bolognesi M, et al. Doppler sonography and hepatic vein catheterization in portal hypertension: assessment of agreement in evaluating severity and response to treatment. J Hepatol 1998; 28: 622-30

33. Schneider AW, Kalk JF, Klein CP. Hepatic arterial pulsatility index in cirrhosis: correlation with portal pressure. J Hepatol 1999; 30; 876-81.

34. Tasu JP, Rocher L, Péletier G, et al. Hepatic venous pressure gradients measured by duplex ultrasound. Clinical Radiology 2002; 57: 746-52.

35. Buonamico P, Sabbá C, García-Tsao G, et al. Octreotide blunts postprandial splachnic hyperemia in cirrhotic patients: a double-blind randomized echo-doppler study. Hepatology 1995; 21: 134-9.

36. Cioni G, D'Alimonte P, Zerbinati F, et al. Duplex-doppler ultrasonography in the evaluation of cirrhotic patients with portal hypertension and in the analysis of their response to drugs. J Gastroenterol Hepatol 1992; 7: 388-92.

37. De la Peña J, Silvan M, Colman T, et al. Estudio hemodinámico y con ecografía doppler en la hipertensión portal. Importancia en el seguimiento de los pacientes tratados con propanolol y mononitrato de isosorbida. Gastroenterología y Hepatología 1994; 17: 112-6.

38. Tincani E, Cioni G, D’Alimonte P, et al. Effects of propanolol compared with clonidine on portal haemodynamics: a double-blind crossover study using duplex-doppler ultrasonography. Eur J Gastroenterol Hepatol 1995; 7: 893-7.

39. Zoli M, Magalotti D, Ghigi G, et al. Transdermal nitroglycerin in cirrhosis. A 24 hour echo-doppler study of splachnic hemodynamics. J Hepatol 1996; 25: 498-503.

40. Tincani E, Cioni G, Cristani A, et al. Duplex doppler ultrasonographic comparison of the effects of propanolol and isosorbide-5-mononitrate on portal haemodynamics. J Ultrasound Med 1993; 12: 525-9.

41. Bolognesi M, Sacerdoti D, Merkel C, et al. Duplex doppler sonographic evaluation of splachnic and renal effects of single agent and combined therapy with nadolol and isosorbide-5-mononitrate in cirrhotic patients. J Ultrasound Med 1994; 13: 945-52.
42. Piscaglia F, Gaiani S, Siringo S, et al. Duplex-doppler evaluation of the effects of propanolol and isosorbide-5-mononitrate on portal flow and splachnic arterial circulation in cirrhosis. Aliment Pharmacol Ther 1998; 12: 475-81.

43. Ohnishi K, Saito M, Nakayama T, et al. Effects of vasopressin on portal hemodynamics in patients with portal hypertension. Am J Gastroenterol 1987; 82: 135-8.

44. Matsutani S, Mizumoto H, Fukuzawa T, et al. Response of blood flow to vasopressin in the collateral left gastric vein in patients with portal hypertension. J Hepatol 1995; 23: 557-62.

45. Iwao $\mathrm{T}$, Toyonaga $\mathrm{A}$, Oho $\mathrm{K}$, et al. Effect of vasopressin on esophageal varices blood flow in patients with cirrosis: comparisons with the effectcs on portal vein and superior mesenteric artery blood flow. J Hepatol 1996; 25 (4): 491-7.

46. Ohnishi K, Sato S. Effects of vasopressin on left gastric venous flow in cirrhotic patients with esophageal varices. Am J Gastroenterol 1990; 85 (3): 293-5.

47. McCormick PA, Seifalian AM, Stansby G, et al. Superior mesenteric artery blood blood flow in man measured with intraarterial doppler catheters: effect of octreotide. J Hepatol 1993; 17: 20-7.

48. D'Alimonte P, Cioni G, Cristani A, et al. Duplex-doppler ultrasonography in the assessment of portal hypertension. Utility of the measurement of maximum portal flow velocity. Eur J Radiol 1993; 17: 126-9.

49. Siringo S, Bolondi L, Gaiani S, et al. The relationship of endoscopy, portal doppler ultrasound flowmetry, and clinical and biochemical tests in cirrhosis. J Hepatol 1994; 20: 11-8.

50. Zoli M, Marchesini G, Brunori A, et al. Portal venous flow in response to acute beta-bloquer and vasodilatatory treatment in patients with liver cirrhosis. Hepatology 1986; 6: 1248-51.

51. Ljubicic N. Effect of metoclopranide on portal blood flow in patients with liver cirrhosis, measured by the pulsed doppler system. Scand J Gastroenterol 1990; 25: 1004-9.

52. Mottet C, Sieber CC, Nauer A, et al. Hemodynamic effects of the somatostatin analogue lanreotide in humans: placebo-controlled, cross-over dose-ranging echo-doppler study. Hepatology 1998; 27: 920-5.

53. Ludwig D, Schadel S, Bruning A, et al. 48-hour hemodynamic effects of octreotide on postprandial splachnic hyperemia in patients with liver cirrhosis and portal hypertension: double-blind, placebocontrolled study. Dig Dis Sci 2000; 45 (5): 1019-27.

54. Saruc M, Can M, Kücükmetin N, et al. Somatostatin infusion and hemodynamic changes in patients with non-variceal upper gastrointestinal bleeding: a pilot study. Med Sci Monit 2003; 9 (7): 184-7. 